\title{
Acquisition of a conditioned aversion as a function of age and measurement technique
}

\author{
STEPHEN B. KLEIN, GARY C. DOMATO, CAROL HALLSTEAD, \\ INGRID STEPHENS, and PETER J. MIKULKA \\ Old Dominion University, Norfolk, Virginia 23508
}

\begin{abstract}
Two experiments investigated variables influencing the acquisition of a conditioned aversion in juvenile and adult rats. In the first experiment, juvenile and adult subjects received six scopolamine-illness pairings utilizing a forced single-bottle measurement technique. Whereas adult rats learned an aversion to sucrose more rapidly when sucrose was novel rather than familiar, neither familiarized nor novel juvenile animals exhibited any aversion to sucrose. The second experiment compared acquisition of a taste aversion in juvenile and adult animals with either a one-bottle forced technique or a two-bottle choice procedure. Adult rats acquired an aversion to sucrose associated with $\mathrm{LiCl}$ illness more rapidly with a two-bottle than with a one-bottle measurement technique, although a strong sucrose aversion was seen in adult rats after four sucrose-illness pairings with the one-bottle technique. Young juvenile rats avoided sucrose when a two-bottle free-choice procedure was employed, while showing only a weak aversion to sucrose with the forced technique after four pairings. These results show that young animals can learn to avoid a taste associated with illness if the appropriate conditions are employed.
\end{abstract}

Animals readily avoid a taste stimulus paired with illness (e.g., Garcia \& Koelling, 1966). Conditioned aversion refers to the phenomenon where an organism associates a taste with subsequent illness, even though the gustatory stimulus may precede illness by several hours.

Since, in a conditioned aversion situation, the rat is required to inhibit consumption of a previously ingested taste, one might expect developmental processes to influence the occurrence of taste aversion. Young animals appear to have more difficulty than adults in passively avoiding an aversive event, where shock is the aversive stimulus (e.g., Riccio, Rohrbaugh, \& Hodges, 1968; Riccio \& Schulenberg, 1969). One purpose of the first experiment was to determine if young juvenile rats would avoid a taste whose consumption preceded illness.

Novelty of the taste solution appears to be an important determinant influencing acquisition of a conditioned aversion (e.g., Garcia \& Koelling, 1967; Revusky \& Bedarf, 1967). Animals develop aversions to novel tastes more rapidly than they do to familiar tastes (e.g., Fenwick, Mikulka, \& Klein, 1975). Kalat and Rozin (1973) have suggested that when illness does not occur after consumption of a particular taste the rat learns that the taste is safe and can be ingested again. Thus, learned safety interferes with the development of aversions to previously experienced tastes.

This research was supported by a grant to the first author by the Old Dominion University Research Foundation. Requests for reprints should be sent to Stephen B. Klein, Department of Psychology, Old Dominion University, Norfolk, Virginia 23508.
Young animals also appear to have more difficulty inhibiting previously appropriate responses than do adults. Egger (1974) found that 24-day-old rats extinguished an acquired escape response more slowly than either 50- or 100-day-old animals. Since, as a consequence of familiarization, the subject learns that a particular taste is safe, young rats may exhibit more interference with taste aversion acquisition when previously experiencing that taste without subsequent illness exposure.

\section{EXPERIMENT I}

The first experiment was designed to investigate whether young animals will develop an aversion to sucrose following sucrose-illness exposures. Juvenile and adult rats received either prior exposure to sucrose or no prior experience with sucrose before sucrose-scopolamine pairings. Acquisition of a conditioned aversion was continued until suppression criterion was reached or animals received six sucrose-illness pairings.

\section{Method}

Subjects. The subjects were $\mathbf{4 8}$ naive female rats purchased from Sprague-Dawley (Madison, Wisconsin). Twenty-four rats were 23 days old (juvenile) and the other 24 rats (adult) were 65 days old at the beginning of the experiment. All rats were individually housed and maintained on ad-lib food.

Procedure. At the beginning of Experiment $\mathrm{I}$, all rats were placed on an ad-lib $\mathrm{H}_{2} \mathrm{O}$ for $24 \mathrm{~h}$, and the amount consumed was measured. Each subject was then given $85 \%$ of the ad-lib water consumption for the next $24 \mathrm{~h}$. Eight juvenile and eight adult rats received $10 \%$ sucrose solution in an amount equal to an $85 \%$ water ration during Days 3-5 of the first experiment. Other animals continued to receive $85 \%$ of the amount of $\mathrm{H}_{2} \mathrm{O}$ consumed on Day 1 on Days 3-5. All rats 


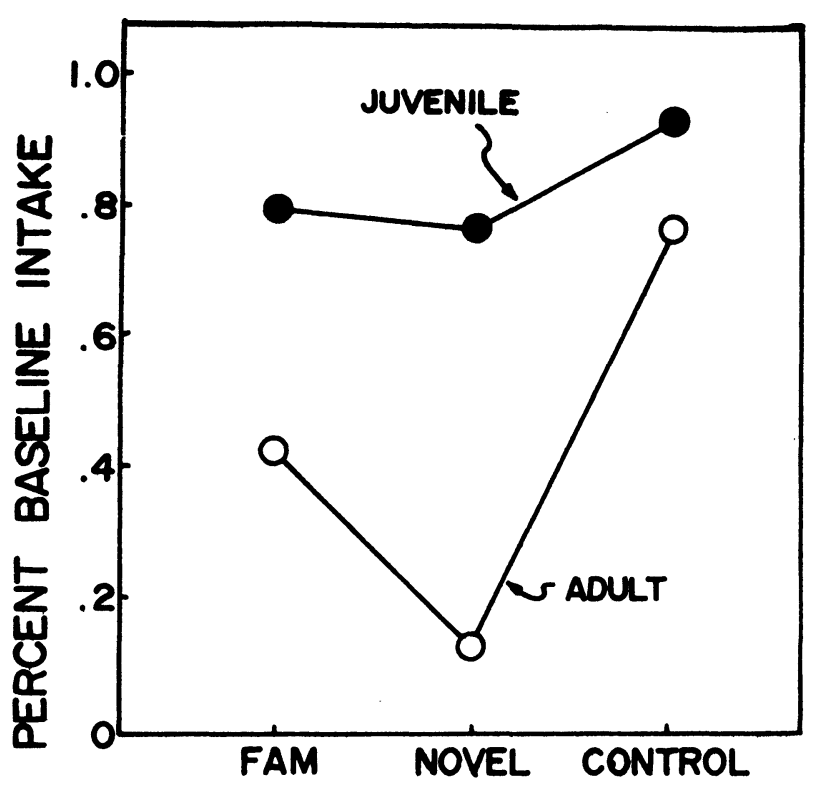

TREATMENTS

Figure 1. Mean percent baseline sucrose water solution intake during six sucrose-scopolamine pairings for familiarized, novel, and control adult and juvenile rats.

then received $10 \%$ sucrose for 15 min on Day 6 . The subjects given sucrose on Days 3-5 (familiar-illness subjects), and half of the animals who received $\mathrm{H}_{2} \mathrm{O}$ on Days 3-5 (novel-illness subjects) were administered a $1-\mathrm{mg} / \mathrm{kg}$ injection of methyl scopolamine (dose level used by Berger, 1972, to induce conditioned aversion) following sucrose ingestion on Day 6 . The remaining eight juvenile and eight adult rats received a saline injection following sucrose exposure on Day 6. Ad-lib water was then made available for the next $23.75 \mathrm{~h}$ to all subjects. Animals received sucrose-illness pairings daily for 6 days, and the influence of sucrose-illness pairings on amount of sucrose consumed was evaluated during the 15-min sucrose exposure on the following day. Testing was discontinued if the rat consumed less than $1 \mathrm{~g}$ of sucrose during the 15-min exposure. A subject's last suppression score was utilized on subsequent days until treatment was discontinued for all subjects after six sucrose-scopolamine pairings.

The percent of first-day consumption was determined for each of the six test days, and an Age by Treatment by Days analysis of variance was performed. Since no days effects were significant, additional data analyses employed a single preference score representing a mean (percent of) baseline consumption score for each subject during 6 test days.

Subjects were placed on deprivation prior to first sucrose-illness pairing to facilitate consumption of sucrose on first taste-illness experience. A nondeprivation testing procedure was employed in an attempt to control for differences in motivational level between juvenile and adult rats created by the deprivation procedure. Subjects not consuming at least $2 \mathrm{~g}$ of $10 \%$ sucrose on Day 6 of this experiment were discarded, and their data were not included in statistical analysis. One juvenile rat in the novel-illness group and two rats in each adult group did not drink $2 \mathrm{~g}$ of sucrose on Day 6.

\section{Results and Discussion}

Amount of $10 \%$ sucrose water consumed $(.1 \mathrm{~g}$ sucrose/.90 $\mathrm{H}_{2} \mathrm{O}$ ) served as the measure of aversion. Analysis of percent baseline fluid intake during testing demonstrated a significant age effect $(\mathrm{F}=74.29, \mathrm{df}=$ $1 / 42, \mathrm{p}<.001)$, a significant treatment effect $(\mathrm{F}=$
$25.24, \mathrm{df}=2 / 42, \mathrm{p}<.001)$, and a significant Age by Treatment interaction $(\mathrm{F}=6.43, \mathrm{df}=2 / 42, \mathrm{p}<.01)$. A Duncan's range test $(p<.01)$ demonstrated that novel-illness adult rats drank significantly less sucrose than did either adult rats familiarized with sucrose prior to sucrose-illness pairing or control adult animals (see Figure 1). The percentage of sucrose consumption in familiarized-illness subjects was significantly less than observed in control nonillness adult rats.

In contrast to the aversion to sucrose demonstrated in adult rats, juvenile rats given sucrose-scopolamine pairings consumed as much sucrose as control rats not receiving scopolamine following sucrose exposure. There were no differences in percent sucrose intake between juvenile familiarized-illness rats, juvenile novel-illness animals, and juvenile nonillness animals. These results indicate that, in contrast to adult rats, juveniles fail to show an avoidance of sucrose following sucrose-illness pairings.

There are several possible explanations for why young rats did not exhibit an aversion to sucrose when paired with illness in the first experiment. It may be that young animals are not able to develop a taste aversion. The passive-avoidance deficits noted in young rats (Riccio, Rohrbaugh, \& Hodges, 1968; Riccio \& Schulenberg, 1969) have been attributed to the slower development of inhibitory systems in rats. Development of an aversion to sucrose when followed by illness may depend upon the development of these inhibitory systems.

However, the results of Klein, Barter, Murphy, and Richardson (1974) demonstrated that although juvenile rats had difficulty maintaining an aversion to sucrose containing mercury, an aversion to sucrose was observed at various times during testing in young rats. These results suggest that the apparent failure of juvenile rats to avoid sucrose in Experiment I may not be due to a general failure to learn to avoid a taste preceding illness but rather some aspect of the situation which precluded the appearance of an aversion to sucrose in juvenile rats. The testing procedure employed in Experiment I involved a single solution being available during testing. Research in conditioned aversion has demonstrated that a single-bottle measurement technique is not the most sensitive index of aversion strength (Dragoin, McCleary, \& McCleary, 1971; Grote \& Brown, 1971a). Animals exhibit a stronger aversion when a two-bottle choice situation is employed. The conflict between thirst and avoidance of a single fluid source present in a single-bottle test but eliminated by the choice measurement technique may be responsible for the observed differences between forced and choice procedures.

Although a nondeprivation procedure was employed in the first experiment, the strong preference for sucrose combined with the inhibitory deficits present in young animals may have been sufficient to obscure 
possible aversion to sucrose in young animals when the forced one-bottle procedure was used. Inhibitory deficits in juvenile animals would influence avoidance less in a two-choice situation, as the young animals would have some other solution to consume instead of sucrose, assuming the juvenile had developed an aversion during sucrose-illness pairings.

\section{EXPERIMENT II}

The purpose of the second experiment was to determine if juvenile rats would develop an aversion to sucrose when a two-bottle choice situation rather than a forced one-bottle measurement procedure was used. Juvenile and adult rats received $\mathrm{LiCl}$ injections following sucrose consumption, with the subsequent aversion to sucrose being evaluated utilizing either a one-bottle or a two-bottle measurement technique.

\section{Method}

Subjects. The subjects were 60 experimentally naive female rats purchased from Sprague-Dawley, Inc. (Madison, Wisconsin). Half of the rats were 23 days old and the other half were 65 days old at the onset of the second experiment. All subjects were individually caged and maintained on an ad-lib food schedule.

Procedure. Upon arrival in the laboratory, all animals received 1 day of ad-lib $\mathrm{H}_{2} \mathrm{O}$. For the remainder of the experiment, all rats were on a 23.5-h water-deprivation schedule. Following 5 days of deprivation, 15 juvenile and 15 adult rats received $15 \mathrm{~min}$ of sucrose followed by 15 min of $\mathrm{H}_{2} \mathrm{O}$. After exposure to sucrose and $\mathrm{H}_{2} \mathrm{O}$ on a forced one-bottle technique, 9 adult and 9 juvenile rats (forced-illness groups) were administered an IP .15 M LiCl solution in an amount equal to $2 \%$ body weight. The other 6 adult and 6 juvenile animals (forced nonillness groups) were given an equal volume $(.5 \mathrm{cc} / 100 \mathrm{~g})$ of bacteriostatic sodium chlorida following 15 min of $\mathrm{H}_{2} \mathrm{O}$.

The remaining 15 juvenile and 15 adult rats were trained utilizing a free two-bottle choice technique. In the free-choice procedure, sucrose and $\mathrm{H}_{2} \mathrm{O}$ were both available during the first $15 \mathrm{~min}$ of training followed by $15 \mathrm{~min}$ of $\mathrm{H}_{2} \mathrm{O}$. Nine adult and 9 juvenile free-choice animals received a $.15 \mathrm{M} \mathrm{LiCl}$ injection after 15 min $\mathrm{H}_{2} \mathrm{O}$ exposure, while the other 6 adult and 6 juvenile rats were administered $\mathrm{NaCl}$ following the free-choice procedure. The free-choice measurement animals were divided into four groups on Day 6: free-illness adult group, free-illness juvenile group, free nonillness adult group, and free nonillness juvenile group. The time interval between taste experience and illness experience was equivalent for free and forced animals; $15 \mathrm{~min}$ after sucrose was removed, $\mathrm{LiCl}$ was administered. Amount of sucrose and $\mathrm{H}_{2} \mathrm{O}$ consumed during testing was recorded. On Day 7 of the second experiment, all animals received 30 min access to $\mathrm{H}_{2} \mathrm{O}$ and subjects did not receive any injection.

On Day 8, free-choice animals received $15 \mathrm{~min}$ exposure to sucrose and $\mathrm{H}_{2} \mathrm{O}$ followed by $15 \mathrm{~min}$ of $\mathrm{H}_{2} \mathrm{O}$, while forced subjects were given $15 \mathrm{~min}$ of sucrose followed by $15 \mathrm{~min}$ of $\mathrm{H}_{2} \mathrm{O}$. After sucrose and $\mathrm{H}_{2} \mathrm{O}$ experience under either the forced or the free measurement technique, illness rats were again administered $\mathrm{LiCl}$ while nonillness rats continued to receive $\mathrm{NaCl}$ injections. Thirty minutes of $\mathrm{H}_{2} \mathrm{O}$ was available to both illness and nonillness animals on Day 10 of training. All subjects continued to received appropriate injections on alternating days until either reaching suppression criterion or the end of the second experiment. Water was available to animals for $30 \mathrm{~min}$ on noninjection days.

Illness subjects received $\mathrm{LiCl}$ injections following access to sucrose until the rat consumed less than $1 \mathrm{~g}$ of sucrose during $15 \mathrm{~min}$ exposure to sucrose. After reaching suppression criterion, each subject was placed on ad-lib water, and its last intake score was utilized on subsequent training days for statistical analysis. Testing was discontinued for all illness rats who had not suppressed after four sucrose-illness pairings and for all nonillness animals after the fifth test day.

\section{Results and Discussion}

Simple analyses of variance were performed on amount of fluid intake (grams) for each day of suppression testing to evaluate the course of the acquisition of the conditioned aversion. Duncan range tests (Edwards, 1960) were employed to determine specific differences within a particular day. Although treatment was not continued for a particular rat once reaching criterion, its last day suppression data was utilized on subsequent analyses until treatment was discontinued for all subjects.

An analysis of the sucrose consumption on Day 1 of training yielded a significant age effect $(F=132.78, \mathrm{df}$ $=1 / 52, \mathrm{p}<.01)$, a significant procedure effect $(\mathrm{F}=$ 11.01 , df $=1 / 52, p<.01$ ), and a significant Age by Procedure interaction $(F=5.08, \mathrm{df}=1 / 52, \mathrm{p}<.05)$. All other effects were nonsignificant on Day 1. Duncan's range tests showed that consumption of sucrose was significantly greater in all adult groups than in any juvenile group. Adult rats receiving the forced measurement procedure drank more sucrose than adult rats in the free-choice groups. There were no other significant sucrose consumption differences between groups on Day 1 of training.

Analysis of water consumption for first test day was also performed. The higher intake of sucrose by adult foced animals than by adult free animals was matched by a corresponding lower intake of $\mathrm{H}_{2} \mathrm{O}$, so that total fluid consumption of Day 1 was equivalent in adult rats. In addition, the juvenile rats did not differ in terms of water intake on Day 1 of training as well as sucrose intake. Procedures inducing higher sucrose consumption produced correspondingly lower water intake, the net effect being that total fluid intake in adult as well as in juvenile rats was the same regardless of whether the free or the forced-choice procedure was used.

Four 2 by 2 by 2 analyses of variance were performed on the sucrose intake data on Days 2-5 of training. Significant age effect $(\mathrm{Fs}=30.19,18.00,6.27,5.76, \mathrm{df}$ $=1 / 52, \mathrm{p}<.05)$, and significant drug effects $(\mathrm{Fs}=$ $83.87,191.09,87.65,251.39, \mathrm{df}=1 / 52, \mathrm{p}<.01$ ) were found on Days 2-5 of training. Analysis of sucrose intake on the second and third test days yielded significant procedure effects $(\mathrm{Fs}=12.75,8.89, \mathrm{df}=$ $1 / 52, \mathrm{p}<.05)$ and significant Procedure by Drug interaction effects $(F s=5.75,7.03$, df $=1 / 52$, $\mathrm{p}<.05$ ). Significant Age by Drug interactions (Fs $=$ $29.23,69.30,44.39, \mathrm{df}=1 / 52, \mathrm{p}<.05$ ) were observed on the second, third, and fourth test days. No other significant effects were found on Days 2-5 of testing. 
Duncan's range test showed that adult free-illness animals drank significantly less sucrose than was observed in nonillness free animals on Days 2-5 of testing, while sucrose consumption was less in adult illness forced rats than was found in adult nonillness forced subjects on each test day (see Figure 2). The two nonillness adult groups drank an equal amount of sucrose on each test day, whereas adult illness groups differed on Test Days 2 and 3 but drank the same amount of sucrose on Test Days 4 and 5 .

As was demonstrated in adult rats, juvenile illness free-choice rats consumed less sucrose than was shown in nonillness free animals on each test day. Illness forced juvenile subjects drank less sucrose than nonillness forced control rats only on Day 5 , while nonillness free and forced rats did not differ on any of the test days. Juvenile free-illness rats consumed less sucrose than forced illness rats on Days 2 and 3 of testing. The data were also analyzed using trials to criterion as the dependent variable, and a similar pattern of results was found.

The results demonstrated that adult illness animals developed an aversion to sucrose as a consequence of sucrose- $\mathrm{LiCl}$ pairing. As in prior research, acquisition of taste aversion was more rapid in the free than in the forced measurement technique. In the juvenile rats, free-choice animals acquired a sucrose-illness association, while forced illness animals differed from forced nonillness control rats only on the last test day. This difference does not represent a decrease in sucrose consumption for illness animals, but rather occurred because of an increase in amount of sucrose drunk by nonillness rats.

Figure 3 presents water consumption data for juvenile and adult rats. Analyses of water intake agree with those obtained with sucrose intake. A number of observations regarding $\mathrm{H}_{2} \mathrm{O}$ intake are important.
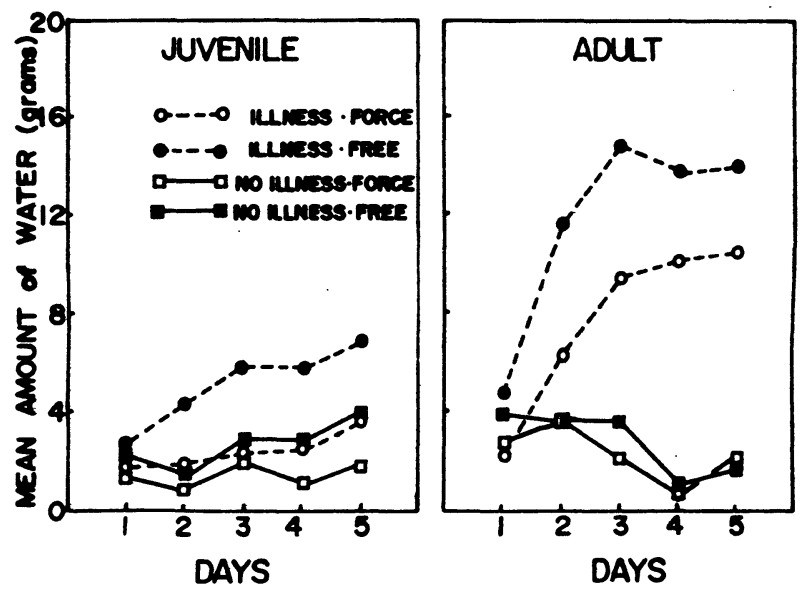

Figure 3. Mean water intake (grams) during acquisition of conditioned aversion as a function of age, drug treatment, and measurement technique.
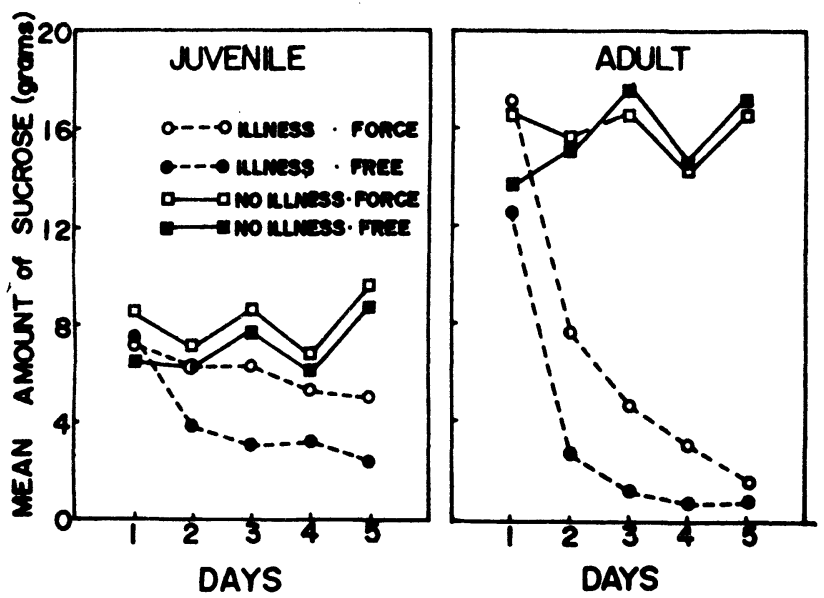

Figure 2. Mean sucrose water solution intake (grams) during acquisition of conditioned aversion as a function of age, drug treatment, and measurement technique.

First, while adult illness rats drank less sucrose than adult nonillness animals on the second through fifth test day, illness animals drank more $\mathrm{H}_{2} \mathrm{O}$. Thus, as the sucrose consumption in illness adult rats declined, water consumption increased. Second, total fluid intake appeared to remain equivalent in all adult rats even though sucrose intake was declining in illness animals. Third, water intake in juvenile illness free-choice rats also increased as sucrose intake decreased. Thus, juvenile illness free animals were able to maintain a total intake equal to nonillness controls in spite of decreased sucrose consumption.

\section{GENERAL DISCUSSION}

As in previous research (Dragoin, McCleary, \& McCleary, 1971; Grote \& Brown, 1971a), acquisition of the conditioned aversion was significantly more rapid in adult rats when a free rather than a forced measurement technique was employed. Consumption of sucrose was significantly lower in adult free illness rats on the second and third test days than observed in adult forced animals. At the end of training, both free and forced adult illness animals had reached a low suppression criterion. Trials to criterion data showed that free-illness animals developed an aversion more quickly than forced-illness rats. Forced illness animals eventually do learn to avoid sucrose when compared with nonillness rats. Both free and forced animals had learned to avoid sucrose as a consequence of sucrose-illness pairings.

The increased $\mathrm{H}_{2} \mathrm{O}$ consumption by adult illness animals over 5 test days indicates that a general malaise induced by $\mathrm{LiCl}$ illness was not responsible for decreased sucrose intake. Adult illness animals were not unable to consume any liquid solution, but rather 
had developed a specific aversion to sucrose and maintained a constant fluid intake by increasing $\mathrm{H}_{2} \mathrm{O}$ consumption.

In the first experiment, juvenile animals failed to exhibit an aversion to sucrose after six sucrosescopolamine pairings. The forced procedure appears to have been responsible for the failure of young animals to avoid sucrose in Experiment I. Juvenile illness free animals drank significantly less sucrose on Days 2 through 5 of testing than corresponding nonillness free animals, while juvenile illness forced animals consumed less sucrose than nonillness forced rats only on Day 5 of testing. The difference in intake between nonillness and illness forced rats on the fifth exposure to sucrose was not due to a decrease in sucrose intake by juvenile illness subjects but rather due to an increase in intake from Days 4 to 5 of testing in juvenile nonillness animals. However, juvenile illness forced animals did exhibit a slight, but significant, decrease in sucrose intake from Day 1 to Day 5 of sucrose exposure $(t=4.14, d f=8, p<.01)$. In terms of trials to criterion results, juvenile free illness animals learned to avoid sucrose, while juvenile forced rats were never able to reach suppression criterion.

The observed minimal aversion to sucrose in forced juvenile rats and strong avoidance of sucrose in free juvenile animals, indicates the importance of measurement technique on acquisition of a conditioned aversion. Acquisition of a sucrose aversion was more rapid in juvenile animals receiving the free rather than the forced measurement procedure. It appears that young animals can avoid a taste associated with illness if appropriate test conditions are employed.

Research seems to indicate that juvenile rats are unable to passively avoid a shock source (e.g., Riccio, Rohrbaugh, Hodges, 1968; Riccio \& Schulenberg, 1969). However, Feigley and Spear (1970) found that although young animals do learn to passively avoid more slowly than adults when identical large shuttleboxes are employed, if the shuttlebox is scaled down to the size of small juvenile rats, acquisition of the passive avoidance was as rapid in young animals as that observed in adult rats. Given appropriate test conditions, young animals can effectively inhibit approach behavior and passively avoid an aversive experience.

It may be that young rats have inhibitory deficits but can learn to avoid an aversive experience. Conditions must be appropriate for the animal to overcome its inhibitory deficiencies and avoid the aversive stimulus. In the case of a conditioned aversion, the opportunity to consume $\mathrm{H}_{2} \mathrm{O}$ rather than sucrose in a free-choice procedure may have enabled the juvenile rat to avoid sucrose and thereby overcome its inhibitory deficits, whereas the combination of a strong approach behavior and an inhibitory deficit may have prevented the young animal from avoiding the sucrose solution for $15 \mathrm{~min}$ and waiting for $\mathrm{H}_{2} \mathrm{O}$ in forced measurement technique.

The young animal has as strong a need to maintain a consistent total intake of fluid as an adult. Adult illness rats increase $\mathrm{H}_{2} \mathrm{O}$ intake to compensate for decreased sucrose consumption in order to maintain a set level of fluid intake. Juvenile animals receiving the free-choice procedure also maintain consistent fluid consumption by increased water intake to offset decreased sucrose consumption. In the forced procedure, the animal has to wait $15 \mathrm{~min}$, while sucrose is available, before $\mathrm{H}_{2} \mathrm{O}$ is presented. It may be very difficult for young animals, having the need to maintain a set level of fluid consumption and inhibitory deficits, to wait $15 \mathrm{~min}$ for $\mathrm{H}_{2} \mathrm{O}$ and avoid sucrose. Even though animals may know sucrose is aversive, forced procedure may prevent a juvenile from avoiding sucrose.

Two studies have found that juvenile rats can learn to avoid a taste following illness experience. Grote and Brown (1971b) found that consumption of $\mathrm{LiCl}$ was lowered in juveniles following one experience with $\mathrm{LiCl}$. Although it is difficult to interpret a single group study, young animals appear to avoid the taste of LiCl. Similarly, the results of Klein, Barter, Murphy, and Richardson (1974) showed that juvenile animals will avoid a sucrose solution containing mercury but have difficulty maintaining that aversion. The results presented in this article indicate that young animals can learn to avoid a taste but that appropriate conditions must exist.

\section{REFERENCES}

Dragoin, W., McCleary, G. E., \& McCleary, P. A. A comparison of two methods of measuring conditioned taste aversion. Behavior Research Methods \& Instrumentation, 1971, 3, 309-310.

EDWARDs, A. L. Experimental design in psychological research. New York: Holt, Rinehart \&Winston, 1960.

EGGER, G. J. Escape learning: Acquisition and extinction rates as a function of age in rats. Developmental Psychobiology, 1974, 7, 281-288.

Feigley, D. A., \& Spear, N. E. Effect of age and punishment condition on long-term retention by the rat of active- and passive-avoidance learning. Journal of Comparative and Physiological Psychology, 1970, 73, 515-526.

Fenwick, S., Mikulka, P. J., \& Klein, S. The effect of pre-exposure to sucrose on the acquisition and extinction of a conditioned aversion. Behavioral Biology, 1975, in press.

Garcia, J., \& Koelling, R. A. Relation of cue to consequence in avoidance learning. Psychonomic Science, 1966, 4. 123-124.

Garcia, J., \& Koelling, R. A comparison of aversion induced by $\mathrm{x}$-rays, drugs, and toxins in the rat. Radiation Research, 1967. (Suppl. 7), 439-450.

Grote, F. W., \& Brown, R. T. Conditioned taste aversions: Two-stimulus tests are more sensitive than one-stimulus tests. Behavior Research Methods \& Instrumentation, 1971, 3, 311-312. (a) 
Grote, F. W., \& Brown, R. T. Rapid learning of passive avoidance by weanling rats: Conditioned taste aversion. Psychonomic Science, 1971, 25, 163-164. (b)

Kalat, J., \& Rozin, P. "Learned safety" as a mechanism in long-delay taste aversion learning in rats. Journal of Comparative and Physiological Psychology, 1973, 83, 198-207.

Klein, S. B., Barter, M. J., Murphy, A. L., \& Richardion, J. H. Aversion to low doses of mercuric chloride in rats. Physiological Psychology, 1974, 2, 397-400.

Revusky, S. H., \& BedARF, E. W. Association of illness with prior ingestion of novel foods. Science, 1967, 155, 219-220.
Riccio, D. C., Rohrbaugh, M., \& Hodges, L. A. Developmental aspects of passive and active avoidance learning in rats. Developmental Psychobiology, 1968, 1, 108-111.

Riccio, D. C., \& Schulenberg, C. J. Age-related deficits in acquisition of a passive avoidance response. Canadian Journal of Psychology, 1969, 23, 429-437.

(Received for publication February 13, 1975; revision received May 9,1975 .) 